\title{
ON F-QUADRATIC STOCHASTIC OPERATORS
}

\author{
U.A. Rozikov ${ }^{1}$, U.U. Jamilov ${ }^{2}$ \\ ${ }^{1}$ Institute of Mathematics, Tashkent, Uzbekistan, e-mail: rozikovu@yandex.ru \\ ${ }^{2}$ College n.43, Romitan, Bukhara region, Uzbekistan. e-mail: jamilovu@yandex.ru
}

\begin{abstract}
In this paper we introduce a notion of $F$ - quadratic stochastic operator. For a wide class of such operators we show that each operator of the class has unique fixed point. Also we prove that any trajectory of the $F$-quadratic stochastic operator converges to the fixed point exponentially fast.

Keywords: Quadratic stochastic operator, Volterra and Non-Volterra operators, simplex.
\end{abstract}

\section{Introduction}

Quadratic stochastic operator frequently arise in many models of mathematical genetics $[1,6-8],[14],[15]$.

The quadratic stochastic operator (QSO) is a mapping of the simplex

$$
S^{m-1}=\left\{x=\left(x_{1}, \ldots, x_{m}\right) \in R^{m}: x_{i} \geq 0, \sum_{i=1}^{m} x_{i}=1\right\}
$$

into itself, of the form

$$
V: x_{k}^{\prime}=\sum_{i, j=1}^{n} p_{i j, k} x_{i} x_{j}, \quad(k=1, \ldots, m),
$$

where $p_{i j, k}$ are coefficients of heredity and

$$
p_{i j, k} \geq 0, \quad \sum_{k=1}^{m} p_{i j, k}=1, \quad(i, j, k=1, \ldots, m) .
$$

Note that each element $x \in S^{m-1}$ is a probability distribution on $E=\{1, \ldots, m\}$.

The population evolves by starting from an arbitrary state (probability distribution on $E$ ) $x \in S^{m-1}$ then passing to the state $V x$ (in the next "generation"), then to the state $V^{2} x$, and so on.

For a given $x^{(0)} \in S^{m-1}$ the trajectory $\left\{x^{(n)}\right\}, n=0,1,2, \ldots$ of $x^{(0)} \in S^{m-1}$ under action QSO (2) is defined by $x^{(n+1)}=V\left(x^{(n)}\right)$, where $n=0,1,2, \ldots$

One of the main problems in mathematical biology consists in the study of the asymptotical behavior of the trajectories. This problem was fully solved for Volterra QSO (see $[6],[8],[12])$ which is defined by (2), (3) and the additional assumption

$$
p_{i j, k}=0, \quad \text { if } k \notin\{i, j\} .
$$


The biological treatment of condition (3) is rather clear: the offspring repeats the genotype of one of its parents.

In paper [6] the general form of Volterra QSO $V: x=\left(x_{1}, \ldots, x_{m}\right) \in S^{m-1} \rightarrow V(x)=$ $x^{\prime}=\left(x_{1}^{\prime}, \ldots, x_{m}^{\prime}\right) \in S^{m-1}$ is given:

$$
x_{k}^{\prime}=x_{k}\left(1+\sum_{i=1}^{n} a_{k i} x_{i}\right)
$$

where $a_{k i}=2 p_{i k, k}-1$ for $i \neq k$ and $a_{k k}=0$. Moreover $a_{k i}=-a_{i k}$ and $\left|a_{k i}\right| \leq 1$.

In papers [6], [8] the theory of QSO (5) was developed using theory of the Lyapunov functions and tournaments. But non-Volterra QSOs (i.e. which do not satisfy the condition (4)) were not in completely studied. Because there is no any general theory which can be applied for investigation of non-Volterra operators. To the best of our knowledge, there are few papers devoted to such operators. Now we shall briefly describe the history of non-Volterra operators.

In [5] it was considered the following family of QSO $V_{\lambda}: S^{2} \rightarrow S^{2}, V_{\lambda}=(1-\lambda) V_{0}+$ $\lambda V_{1}, 0 \leq \lambda \leq 1$, where $V_{0}(x)=\left(x_{1}^{2}+2 x_{1} x_{2}, x_{2}^{2}+2 x_{2} x_{3}, x_{3}^{2}+2 x_{1} x_{3}\right)$ is Volterra operator, $V_{1}(x)=\left(x_{1}^{2}+2 x_{2} x_{3}, x_{2}^{2}+2 x_{1} x_{3}, x_{3}^{2}+2 x_{1} x_{2}\right)$ is non-Volterra operator. Note that behavior of the trajectories of $V_{0}$ is very irregular ([16], [17]).

Also well known the class of bistochastic QSOs which contains Volterra operators and non-Volterra operators as well. In [7], [13] the class of such operators is described.

In [3] a class of kvazi-Volterra operators is introduced. For such operators the condition (4) is not satisfied only for very few values of $i, j, k$.

Papers [9-11] are devoted to study of non-Volterra operators which are generated from Volterra operators (5) by a cyclic permutation of coordinates i.e. $V_{\pi}: x_{\pi(i)}^{\prime}=$ $x_{i}\left(1+\sum_{k=1}^{m} a_{i k} x_{k}\right), i=1, \ldots, m$, where $\pi$ is a cyclic permutation on the set of indices $E$.

Note that each quadratic operator $V$ can be uniquely defined by a cubic matrix $\mathbf{P} \equiv$ $\mathbf{P}(V)=\left\{p_{i j, k}\right\}_{i, j, k=1}^{n}$ with condition (3). Usually (see e.g. [1], [3], [5]-[15], [17], [18]) the matrix $\mathbf{P}$ is known. In [2], [4] a constructive description of $\mathbf{P}$ is given. This construction depends on a probability measure $\mu$ which is given on a fixed graph $G$ and cardinality of a set of cells (configurations) which can be finite or continual. In [2] it was proven that the QSO constructed by the construction is Volterra if and only if $G$ is a connected graph.

In [16] using the construction of QSO for the general finite graph and probability measure $\mu$ (here $\mu$ is product of measures defined on maximal subgraphs of the graph $G$ ) a class of non-Volterra QSOs is described. It was shown that if $\mu$ is given by the product of the probability measures then corresponding non-Volterra operator can be reduced to $N$ number (where $N$ is the number of maximal connected subgraphs) of Volterra operators defined on the maximal connected subgraphs.

Recently by the authors of this paper a new class of non-Volterra operators is intro- 
duced. These operators satisfy

$$
p_{i j, k}=0, \text { if } k \in\{i, j\} .
$$

Such an operator is called strictly non-Volterra QSO. For arbitrary strictly non-Volterra QSO defined on $S^{2}$ we proved that every such operator has unique fixed point. Also it was proven that such operators has a cyclic trajectory and almost all trajectories converge to the cyclic trajectory. This is quite different behavior from the behavior of Volterra operators, since Volterra operator has no cyclic trajectories.

In this paper we consider an other type of non-Volterra operators. Which we call $F-$ quadratic stochastic operators. For a family of such operators we show that each of them has unique fixed point and all trajectories tend to this fixed point exponentially fast. Thus they are ergodic operators.

\section{Definition of $F-$ QSO}

In this paper we extend the set $E$ by adding element " 0 " i.e. we consider $E_{0}=\{0,1, \ldots, m\}$. Fix a set $F \subset E$ and call this set the set of "females" and the set $M=E \backslash F$ is called the set of "males". The element 0 will play the role of "empty-body".

Coefficients $p_{i j, k}$ of the matrix $\mathbf{P}$ we define as follows

$$
p_{i j, k}=\left\{\begin{array}{l}
1, \quad \text { if } k=0, i, j \in F \cup\{0\} \quad \text { or } i, j \in M \cup\{0\} ; \\
0, \text { if } k \neq 0, i, j \in F \cup\{0\} \text { or } i, j \in M \cup\{0\} ; \\
\geq 0, \text { if } i \in F, j \in M, \forall k .
\end{array}\right.
$$

Biological treatment of the coefficients (7) is very clear: a "child" $k$ can be generated if its parents are taken from different classes $F$ and $M$. In general, $p_{i j, 0}$ can be strictly positive for $i \in F$ and $j \in M$, this corresponds, for example, to the case when "female" $i$ with "male" $j$ can not generate a "child" since one of them (or both are) is ill.

Definition 1. For any fixed $F \subset E$, the $Q S O$ defined by (2),(3) and (7) is called $F-$ quadratic stochastic operator.

Remarks. 1. Any $F-$ QSO is non- Volterra since $p_{i i, 0}=1$ for any $i \neq 0$.

2. Note that the class of $F-$ QSOs for a given $F$ does not intersect with the classes of non-Volterra QSOs mentioned above (in Introduction).

3. For $m=1$ there is unique $F-$ QSO (independently on $F=\{1\}$ and $F=\emptyset$ ) which is constant i.e. $V(x)=(1,0)$ for any $x \in S^{1}$.

\section{$3 \quad F-$ QSO for $m=2$}

In this section we consider $m=2$, i.e. $E_{0}=\{0,1,2\}$. Take $M=\{1\}$ and $F=\{2\}$. We will generalize the result of this section in the next section for $m \geq 3$ there $F$ is taken as 
$F=\{2,3, \ldots, m\}$. Reasons of considering the case $m=2$ in a separate section are: (i) in case $m=2$ the number of "females" is equal to the number of "males"; (ii) In this case all calculations can be done explicitly.

For $m=2$, and $M=\{1\}, F=\{2\}$ the $F$-OSO is defined by matrix

$$
\left(\begin{array}{cccccc}
p_{00,0}=1 & p_{01,0}=1 & p_{02,0}=1 & p_{11,0}=1 & p_{12,0}=a & p_{22,0}=1 \\
p_{00,1}=0 & p_{01,1}=0 & p_{02,1}=0 & p_{11,1}=0 & p_{12,1}=b & p_{22,1}=0 \\
p_{00,2}=0 & p_{01,2}=0 & p_{02,2}=0 & p_{11,2}=0 & p_{12,2}=c & p_{22,2}=0
\end{array}\right)
$$

where

$$
a, b, c \geq 0, \quad a+b+c=1
$$

The corresponding $F-$ QSO has the form

$$
V_{0}:\left\{\begin{array}{l}
x_{0}^{\prime}=x_{0}^{2}+x_{1}^{2}+x_{2}^{2}+2 x_{0} x_{1}+2 x_{0} x_{2}+2 a x_{1} x_{2}=1-2(1-a) x_{1} x_{2}, \\
x_{1}^{\prime}=2 b x_{1} x_{2}, \\
x_{2}^{\prime}=2 c x_{1} x_{2} .
\end{array}\right.
$$

The fixed point of $V$ is defined as a solution of the equation $V(x)=x$.

The following theorem completely describes the behavior of the trajectories of operator $(9)$.

Theorem 1. 1) For any a,b,c with condition (8) the operator (9) has unique fixed point $(1,0,0)$.

2) For any $x^{(0)} \in S^{2}$ the trajectory $\left\{x^{(n)}\right\}$ tends to the fixed point $(1,0,0)$ exponentially fast.

Proof. 1) It is easy to see that the solutions of the equation $V_{0}(x)=x$ are only $x=e_{1}=(1,0,0)$ and $x=x^{*}=\left(x_{0}^{*}, x_{1}^{*}, x_{2}^{*}\right)=\left(\frac{2 b c-b-c}{2 b c}, \frac{1}{2 c}, \frac{1}{2 b}\right)$ if $b c \neq 0$ and only $x=e_{1}$ if $b c=0$. Note that $x^{*} \notin S^{2}$. Indeed from $0 \leq \frac{1}{2 b} \leq 1$ and $0 \leq \frac{1}{2 c} \leq 1$ we get $b \geq \frac{1}{2}$ and $c \geq \frac{1}{2}$. Then by (8) we have $b=c=\frac{1}{2}$ i.e. $x_{1}^{*}=x_{2}^{*}=1$ this is impossible since $x_{0}^{*}+x_{1}^{*}+x_{2}^{*}=1$.

2) For $x \in S^{2}$ denote $\varphi(x)=x_{1} x_{2}$. We have

$$
\varphi\left(x^{(n)}\right)=\left\{\begin{array}{l}
0, \quad \text { if } \quad b c=0, \\
(2 b c)^{-1}\left(4 b c x_{1} x_{2}\right)^{2^{n}}, \quad \text { if } \quad b c \neq 0 .
\end{array}\right.
$$

Now we shall estimate $4 b c x_{1} x_{2}$. We have

$$
0 \leq 4 b c x_{1} x_{2} \leq 4 \frac{(b+c)^{2}}{4} \cdot \frac{\left(x_{1}+x_{2}\right)^{2}}{4} \leq \frac{1}{4} .
$$

From the second equality of (9) we get

$$
x_{1}^{(n)}=2 b \varphi\left(x^{(n-1)}\right) .
$$


By (9)-(12) we have

$$
\lim _{n \rightarrow \infty} x_{1}^{(n)}=\lim _{n \rightarrow \infty} x_{2}^{(n)}=0
$$

This completes the proof.

\section{The case $m \geq 2$}

In this section we generalize Theorem 1, but now we have not an explicit formula for $\varphi\left(x^{(n)}\right)$.

Consider $E_{0}=\{0,1, \ldots, m\}, M=\{1\}, F=\{2, \ldots, m\}$. Then it is easy to see that corresponding $F$-QSO has the form (cf. with (9))

$$
V_{1}:\left\{\begin{array}{l}
x_{0}^{\prime}=1-2 x_{1} \sum_{i=2}^{m}\left(1-a_{i 0}\right) x_{i}, \\
x_{k}^{\prime}=2 x_{1} \sum_{i=2}^{m} a_{i k} x_{i}, \quad k=1,2, \ldots, m,
\end{array}\right.
$$

where

$$
a_{i k}=p_{1 i, k} \geq 0, \quad i=2, \ldots, m ; \quad k=0,1, \ldots, m ; \quad \sum_{k=0}^{m} a_{i k}=1, \quad \forall i=2, \ldots, m .
$$

Theorem 2. For any values of $a_{i k}$ with (14) the operator (13) has unique fixed point $(1,0,0, \ldots, 0)$ (with $m$ zeros). Moreover for any $x^{(0)} \in S^{m}$ its trajectory $\left\{x^{(n)}\right\}$ tends to the fixed point exponentially fast.

Proof. For $x \in S^{m}$ denote

$$
\varphi(x)=x_{1} \sum_{i=2}^{m} x_{i}=x_{1}\left(1-x_{0}-x_{1}\right) .
$$

Using (13),(14) we get

$$
x_{k}^{(n+1)}=2 x_{1}^{(n)} \sum_{i=2}^{m} a_{i k} x_{i}^{(n)} \leq 2 x_{1}^{(n)} \sum_{i=2}^{m} x_{i}^{(n)}=2 \varphi\left(x^{(n)}\right),
$$

where $k=1,2, \ldots, m$.

Now we shall estimate $\varphi\left(x^{(n+1)}\right)$ :

$$
\begin{gathered}
\varphi\left(x^{(n+1)}\right)=x_{1}^{(n+1)}\left(1-x_{0}^{(n+1)}-x_{1}^{(n+1)}\right)= \\
\left(2 x_{1}^{(n)} \sum_{i=2}^{m} a_{i 1} x_{i}^{(n)}\right)\left(2 x_{1}^{(n)} \sum_{i=2}^{m}\left(1-a_{i 0}\right) x_{i}^{(n)}-2 x_{1}^{(n)} \sum_{i=2}^{m} a_{i 1} x_{i}^{(n)}\right) .
\end{gathered}
$$

Using AM-GM inequality from (17) we get

$$
\varphi\left(x^{(n+1)}\right) \leq 4\left(x_{1}^{(n)}\right)^{2}\left(\frac{\sum_{i=2}^{m}\left(1-a_{i 0}\right) x_{i}^{(n)}}{2}\right)^{2} \leq\left(x_{1}^{(n)} \sum_{i=2}^{m} x_{i}^{(n)}\right)^{2}=\left(\varphi\left(x^{(n)}\right)\right)^{2}, \quad n \geq 0 .
$$


Note that $\varphi\left(x^{(0)}\right) \leq \frac{1}{4}$. Thus from (18) we obtain

$$
\varphi\left(x^{(n)}\right) \leq\left(\frac{1}{4}\right)^{2^{n}}
$$

Now it follows from (16) and (19) that

$$
\lim _{n \rightarrow \infty} x_{k}^{(n)}=0, \text { for any } k=1,2, \ldots, m,
$$

i.e.

$$
\lim _{n \rightarrow \infty} x^{(n)}=(1,0, \ldots, 0) \text {, for any } x^{(0)} \in S^{m} .
$$

Obviously, $(1,0, \ldots, 0)$ is a unique fixed point, since $(20)$ holds for any $x^{(0)} \in S^{m}$. This completes the proof.

Remarks. 1. The QSO $V$ satisfies the ergodic theorem if the limit

$$
\lim _{n \rightarrow \infty} \frac{1}{n} \sum_{j=0}^{n-1} x^{(j)}
$$

exists for any $x^{(0)} \in S^{m}$. On the basis of numerical calculations Ulam conjectured [15], [17], that the ergodic theorem holds for any QSO. In [18] it was proven that this conjecture is false in general. From Theorem 2 follows that the ergodic theorem holds for any $F-$ QSO determined by (13).

2. Assume a matrix $\mathbf{P}$ with coefficients (7) is given. Let $N_{1}=N_{1}(\mathbf{P})=\left|\left\{i j: p_{i j, 0}=1\right\}\right|$ be the number of 1 in the first row of the matrix $\mathbf{P}$, where $|A|$ denotes the number of elements (cardinality) of $A$. Also denote by $\tilde{N}_{1}=\tilde{N}_{1}(\mathbf{P})$ the number of elements of the first row of $\mathbf{P}$ which $<1$. It is easy to see that $\tilde{N}_{1}=\frac{|E|}{2}(|E|+3)+1-N_{1}$. By (7) one can see that

$$
\begin{gathered}
N_{1} \geq \frac{1}{2}\left(|F|^{2}+|M|^{2}+3|E|\right)+1 \\
\tilde{N}_{1} \leq|F| \cdot|M|
\end{gathered}
$$

Obviously,

$$
\frac{1}{2}\left(|F|^{2}+|M|^{2}+3|E|\right)+1>|F| \cdot|M| \text {. }
$$

Hence $N_{1}>\tilde{N}_{1}$, for any $F \subset E$ and any $\mathbf{P}$ with elements (7). By this property of the matrix $\mathbf{P}$ one can say that 0 has more priority than other elements of $E_{0}$. Thus we can make a

Conjecture. An analogue of Theorem 2 is true for any $F-Q S O$ i.e. for every $F \subset E$, and $\mathbf{P}$ with elements (7).

3. The $F$-QSOs with $F \neq\{2,3, \ldots, m\}$ will be considered in a separate paper. 
Acknowledgments. A part of this work was done within the scheme of Junior Associate at the ICTP, Trieste, Italy and the first author (UAR) thanks ICTP for providing financial support and all facilities (in May - August 2006). The final part of this work was done at the IHES, Bures-sur-Yvette, France. UAR thanks the IHES for support and kind hospitality (in October - December 2006). We also gratitude to professor R. N. Ganikhodzhaev for many helpful discussions.

\section{References}

1. Bernshtein S.N., Solution of a mathematical problem connected with the theory of heredity, Uch. Zap. Nauchno-Issled. kaf. Ukr. Otd. Mat., 1 : 83-115 (1924).

2. Ganikhodjaev N.N., An application of the theory of Gibbs distributions to mathematical genetics, Doklady Math. 61: 321-323 (2000).

3. Ganikhodjaev N.N., Mukhitdinov R.T., On a class of non-Volterra quadratic operators, Uzbek Math. Jour. No. 3-4: 65-69 (2003).

4. Ganikhodjaev N.N., Rozikov U.A., On quadratic stochastic operators generated by Gibbs distributions, Regular and Chaotic Dynamics. 11: No. 3 (2006).

5. Ganikhodzhaev, R. N. A family of quadratic stochastic operators that act in $S^{2}$. Dokl. Akad. Nauk UzSSR. No. 1: 3-5 (1989).

6. Ganikhodzhaev R.N., Quadratic stochastic operators, Lyapunov functions and tournaments, Russian Acad. Sci. Sbornik Math. 76: 489-506 (1993).

7. Ganikhodzhaev, R. N. On the definition of quadratic bistochastic operators. Russian Math. Surveys. 48, no. 4: 244-246 (1992).

8. Ganikhodzhaev R.N., A chart of fixed points and Lyapunov functions for a class of discrete dynamical systems. Math. Notes 56: 1125-1131 (1994).

9. Ganikhodzhaev, R. N.; Dzhurabaev, A. M. The set of equilibrium states of quadratic stochastic operators of type $V_{\pi}$. Uzbek. Mat. Zh. No. 3: 23-27 (1998).

10. Ganikhodzhaev, R. N.; Karimov, A. Z. On the number of vertices of a polyhedron of bistochastic quadratic operators. Uzbek. Mat. Zh. No. 6: 29-35 (1999).

11. Ganikhodzhaev, R. N.; Abdirakhmanova, R. E. Description of quadratic automorphisms of a finite-dimensional simplex. Uzbek. Mat. Zh. No. 1: 7-16 (2002).

12. Ganikhodzhaev, R.N., Eshmamatova D.B. Quadratic automorphisms of simplex and asymptotical behavior of their trajectories. Vladikavkaz Math. Jour. 8: 12-28 (2006).

13. Ganikhodzhaev, R. N.; Eshniyazov, A. I. Bistochastic quadratic operators. Uzbek. Mat. Zh. No. 3: 29-34 (2004).

14. Kesten H. Quadratic transformations: a model for population growth I, II. Adv. Appl. Prob. No.2: 1-82 and 179-228 (1970).

15. Lyubich Yu.I. Mathematical structures in population genetics. Biomathematics, 22, Springer-Verlag, 1992.

16. Rozikov U.A., Shamsiddinov N.B. On non-Volterra quadratic stochastic operators 
generated by a product measure. ICTP preprint 2006, and arXiv:math.DS/0608201.

17. Stein P.R., Ulam S.M. Nonlinear transformations studies on electronic computers. Rozprawy Mat. 39: 1-15 (1964).

18. Zakharevich M.I., The behavior of trajectories and the ergodic hypothesis for quadratic mappings of a simplex. Russian Math. Surveys, 33: 207-208 (1978). 